\title{
Characterisation of internal morphologies in electrospun fibers by X-ray tomographic microscopy
}

\author{
Jens Vinge Nygaard, ${ }^{* a c}$ Tamer Uyar, ${ }^{* b c}$ Menglin Chen, ${ }^{c}$ Peter Cloetens, ${ }^{d}$ Peter Kingshott ${ }^{c f}$ \\ and Flemming Besenbacher ${ }^{c e}$
}

Received 22nd March 2011, Accepted 8th July 2011

DOI: 10.1039/c1nr10304k

\begin{abstract}
Electrospun fabrics for use in, for example, tissue engineering, wound dressings, textiles, filters and membranes have attracted a lot of attention due to their morphological nanoscale architectures which enhance their physical properties. A thorough detailed internal morphological study has been performed on electrospun polystyrene (PS) fibers produced from dimethylformamide (DMF) solutions. Investigations by transmission electron microscopy (TEM) and thorough studies for the first time by synchrotron based $\mathrm{X}$-ray tomographic microscopy (XTM) revealed that the individual electrospun PS fibers and beads have a graded density and in some cases even an internal porous structure.
\end{abstract}

\section{Introduction}

Electrospinning has emerged as a versatile and cost effective technique for producing fibers with micrometre and nanometre diameters. ${ }^{1,2}$ The technique allows for the production of multi-functional nanofibers made from various natural or synthetic polymers, polymer blends and composite solutions, ${ }^{2,3}$ and therefore provides a high degree of flexibility for designing nanofibers with built-in chemical/ physical surface properties. It has been shown that it is indeed the morphological nanoscale architecture of such nanofibers/nanowebs that enhances their properties, and subsequently makes them a highly versatile platform for a broad range of applications in biotechnology, filtration, textile, nanocomposite materials, etc. ${ }^{2-6}$

The response of a material to an applied stimulus is inherently three-dimensional. Morphological property measurements, however,

${ }^{a}$ Aarhus School of Engineering ( ASE), Aarhus University, Ny Munkegade, 8000 Aarhus C, Denmark. E-mail: jvn@iha.dk; Fax: +458942 3690; Tel: $+4541893170$

${ }^{b}$ UNAM-Institute of Materials Science and Nanotechnology, Bilkent University, Bilkent, Ankara, 06800, Turkey. E-mail: tamer@unam. bilkent.edu.tr; Fax: +90 312 2664365; Tel: +90 3122903571

'Interdisciplinary Nanoscience (iNANO), Aarhus University, Ny Munkegade, 8000 Aarhus C, Denmark

${ }^{d}$ European Synchrotron Radiation Facility (ESRF), BP 220, F-38043 Grenoble Cedex, France

${ }^{e}$ Department of Physics and Astronomy, Aarhus University, $N y$ Munkegade, 8000 Aarhus C, Denmark

${ }^{f}$ Industrial Research Institute Swinburne (IRIS), Faculty of Engineering and Industrial Sciences, Swinburne University of Technology, Hawthorn, 3122 VIC, Australia traditionally rely upon two-dimensional analytical techniques, such as scanning electron microscopy (SEM) and transmission electron microscopy (TEM) to explain what three-dimensional responses are, in reality. Analytical tools that have a spatial resolution at the nanometre scale are indispensable for the life and physical sciences. It is desirable that these tools also permit elemental and chemical identification on a scale of $10 \mathrm{~nm}$ or less, with large penetration depths. It has long been known that the linear X-ray attenuation coefficient is a sensitive measure of atomic composition and density. Since the X-ray attenuation coefficient could be measured as a function of position in a sample nondestructively, a variety of techniques $^{7-12}$ in X-ray imaging are currently being developed that may provide these combined capabilities. A spatial resolution of sub-15 $\mathrm{nm}$ has been recently achieved with a soft X-ray microscope. ${ }^{13}$

Here, we have carried out detailed internal morphological studies of electrospun polystyrene (PS) fibers by using synchrotron based X-ray tomographic microscopy (XTM) ${ }^{14,15}$ an analytical method delineates the method as a form of X-ray microscopy that uses tomographic reconstruction techniques to build three dimensional images of micro/nanostructures. Although initial SEM imaging shows that the PS fibers, which are electrospun from dimethylformamide (DMF) solutions, have a nonporous surface. Our detailed TEM and XTM studies, interestingly, reveal significant density variations and in some cases even internal porous regions within the individual fibers. We show that XTM is indeed a very powerful technique to reveal the internal morphology within individual electrospun fibers.

\section{Experimental}

The details of the electrospinning of the polystyrene (PS) fibers have been reported in our previous study. ${ }^{16}$ In short, homogeneous and clear polymer solutions were prepared by dissolving PS ( $M_{\mathrm{w}} \approx 280000$, Sigma-Aldrich) in $N, N$-dimethylformamide (DMF) ( $99 \%$, Sigma-Aldrich) at room temperature. The PS concentration was varied from 10 to $25 \%(\mathrm{w} / \mathrm{v})$ in DMF with the fibers having diameter within the range of $200 \mathrm{~nm}$ to 3 micron were obtained depending on the concentration of the PS solution used. The electrospinning has been carried out at room temperature and the parameters were as follows: the applied voltage was $15 \mathrm{kV}$, the tip-tocollector distance was $10 \mathrm{~cm}$ and the flow rate of polymer solution was $1 \mathrm{ml} \mathrm{h}^{-1}$. A grounded stationary rectangular metal collector 
covered by a piece of aluminium foil was used for the fiber deposition. The collected fibers were dried at $40{ }^{\circ} \mathrm{C}$ in a vacuum oven for $24 \mathrm{~h}$ to remove any residual solvent.

The morphology of the PS fibers was initially studied by means of scanning electron microscopy (SEM) (FEI, Nova 600 NanoSEM). Transmission electron microscope (TEM) images were collected from fibers positioned on a $\mathrm{Cu}$ grid by using a Philips CM20 TEM instrument operated at $200 \mathrm{kV}$.

The X-ray tomographic microscopy (XTM) studies were carried out at the experimental station ID19 of the European Synchrotron Radiation Facility (Grenoble, France) taking advantage of the high contrast acquired due to the high brilliance of the X-ray source realized by the synchrotron. A wiggler was used to select $20 \mathrm{keV}$ photons and by the detection device used, visible light was produced by an X-ray-sensitive converter and imaged onto a cooled chargecoupled device in a FReLoN camera. The end resolution is governed by the beam characteristics (here parallel and not divergent) and the effective pixel size of the detector is selected to be $0.28 \mu \mathrm{m}$ and with a field of view of $0.6 \times 0.6 \mathrm{~mm}^{2}$. The dataset from a single sample is comprised of an angular scan of 1500 images obtained during rotation of the specimen in steps of $0.12^{\circ}$ at a sampledetector distance of $8 \mathrm{~mm}$. The exposure time was $0.15 \mathrm{~s}$ per picture. This image acquisition protocol is followed by a calculation of the spatial distribution of matter reconstructed from the projection images using the parallel beam filtered back-projection algorithm implemented in the ESRF software PyHST. This provides a 3D image comprised of voxels having grayscale values from 0 to 255 . From this image a rendering of the material morphology can be visualized as isosurfaces by interpolating a surface between equivalued voxels. A single voxel within this image has the dimensions of $280 \times 280 \times 280 \mathrm{~nm}$. Thus, slices through this $3 \mathrm{D}$ image can reveal the different densities of the analyzed fibers, and from the differences in densities we can determine the individual fiber morphology. Within this reproduction scheme described above, dark zones corresponded to less dense structures. Webs of electrospun fibers of approximately $8 \mathrm{~mm}^{3}$ were analyzed. They were mounted on the surface of an aluminium cylinder with cyanoacrylate glue, and the cylinder was placed on a rotation stage within the tomography station.

The XTM image processing and the volume renderings were determined using isosurface representations and volume texture renderings generated by the Amira software from Mercury Computer Systems (Chelmsford, MA). Quantitative calculations on the image processed data are performed with MatLab from MathWorks (Natick, MA).

Noise within the original XTM dataset was delimited by use of an edge preserving smoothing filter similar to a Gaussian filter. It smoothes out the difference between gray levels of neighboring voxels and can be interpreted as a diffusion process in which energy between voxels of high and low energy (gray value) is leveled. In contrast to the Gaussian filter method, it does not smear out the edges because the diffusion is reduced or stopped in the vicinity of edges, and thus the absorption edges are preserved. The Amira implementation of this filter with a stop time of 25 in steps of 5 was applied, and the contrast was set at 3.5. In order to make the diffusion process more stable, the image is pre-filtered by a Gaussian filter with the parameter sigma equivalent to 3 voxels $(840 \mathrm{~nm})$. This allows noise to be removed from the image but care must be taken since too large sigma values may remove relevant features.

\section{Results and discussion}

When fibers are electrospun from a polymer solution, the solvent is one of the key parameters which has a significant effect on the fiber morphology, since viscosity, conductivity and surface tension of the polymer solutions depend heavily upon the type of solvent used. ${ }^{1}$ Studies have shown that PS fibers can be electrospun by using different solvent systems such as DMF, tetrahydrofuran (THF), DMF/THF mixture, chloroform, etc. ${ }^{16-18}$ When THF or a mixture of THF/DMF is used as a solvent, ribbon shaped PS fibers with nanoand micro-sized pores on the surface are obtained due to the fast evaporation of the low boiling point THF. Detailed SEM studies show that DMF was the most favorable solvent for producing uniform and round PS fibers with nonporous surfaces due to the high boiling point of DMF as compared to other solvents. ${ }^{16-18}$ We have previously studied the electrospinning of PS in DMF solutions which resulted in beaded and/or bead-free fibers where the fiber diameter varies between $200 \mathrm{~nm}$ and 3 microns and final morphology of the fibers depends on the PS concentration. ${ }^{16}$ Beaded structures were obtained at lower PS concentrations (10\% through 20\% (w/v)) but increasing the PS concentration resulted in a reduced bead formation corresponding to the higher viscosity. At a PS concentration of $25 \%$ (w/v) electrospun fibers having fiber diameter around 1 to 3 micron with a minimal number of beads were obtained.

Initial SEM studies show that the surface of the apparently smooth and nonporous electrospun PS fibers had certain inhomogeneities (Fig. 1). Subsequent TEM images of a PS fiber and a bead are shown in Fig. 2. By enlarging the rectangular marked areas in Fig. 2a and $\mathrm{b}$ and plotting the gray scaled TEM images in color, the heterogeneous structure of both the bead (Fig. 2c) and the fiber (Fig. 2d) is enhanced. Plots of the grayscale distribution across the fiber (Fig. 2f) and the bead (Fig. 2e) demonstrate significant fluctuations, indicating an internal heterogeneous nanostructure. In theory, a TEM image is a projection of the shadow from a specimen illuminated by an e-beam. The grayscale value in a TEM image correlates with the distance that the beam must travel through in the specimen. For

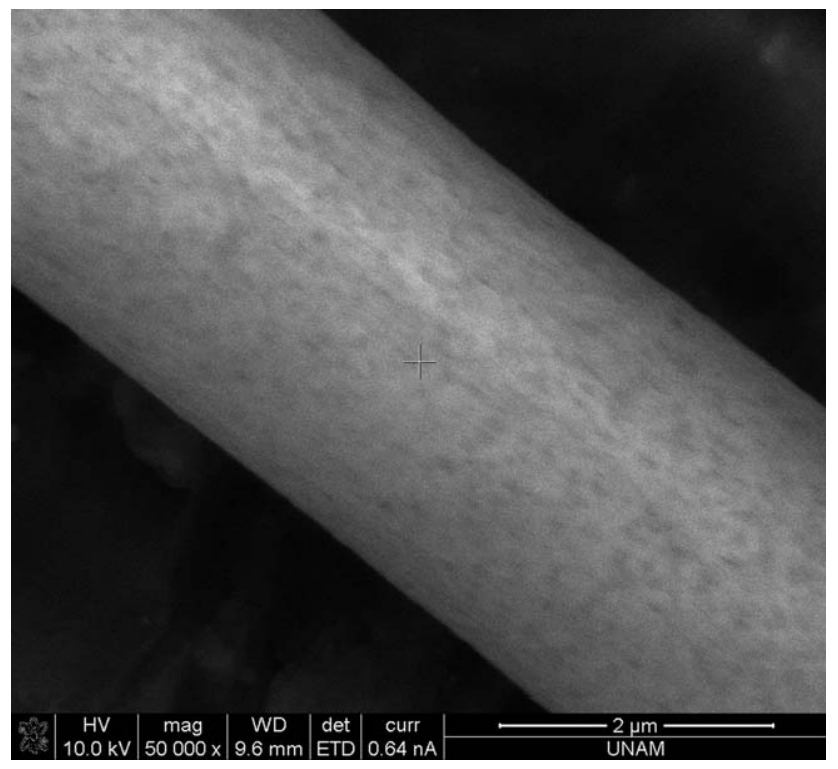

Fig. 1 SEM image of electrospun PS fibers obtained from 25\% (w/v) PS solutions in DMF. 
(a)

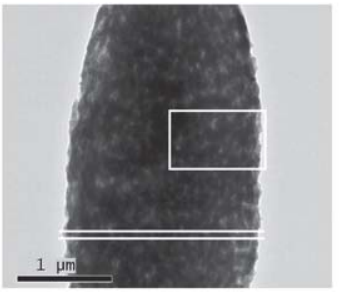

(c)
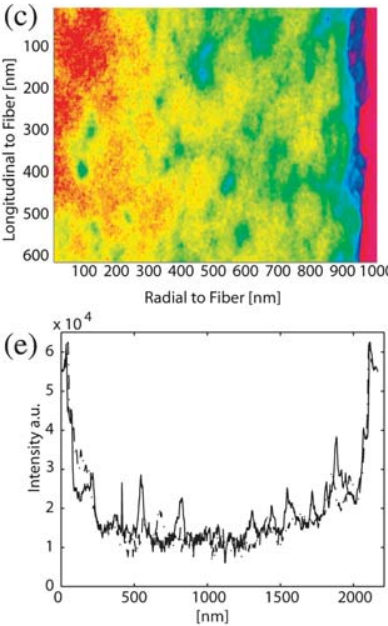

(b)
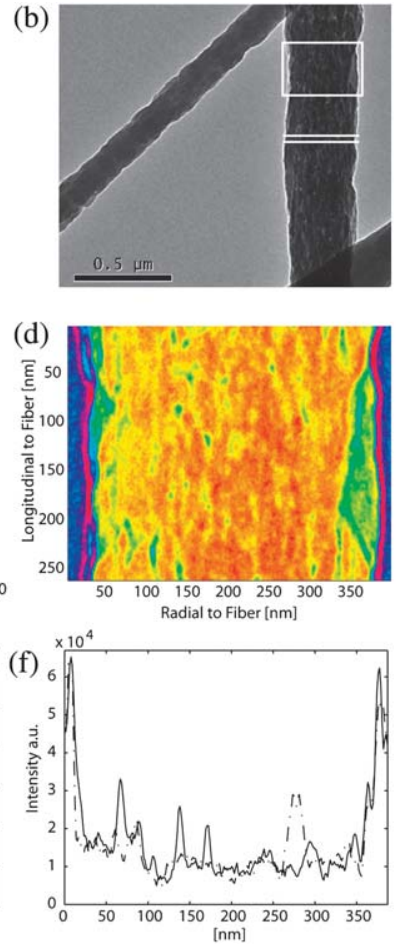

Fig. 2 TEM images of a PS bead (a, c and e) and fiber (b, d and f) obtained from an electrospun PS solution in DMF at a concentration of $10 \%(w / v)$. Two rectangular areas shown in (a) and (b) are enlarged in (c) and (d). Here the grayscale in transformed into a color scale to enhance density variations. The intensity along four cross-sections in (a) and (b) is plotted in (e) and (f) quantifying the density variation across bead and fiber respectively. (e) and (f) show an overall change in image intensity arising from the curvature of the bead and fiber and minor fluctuation arising from density variations through the thickness of the samples.

a perfect homogeneous and round specimen, the shift in grayscale value changes smoothly across it according to its diameter. For a heterogeneous specimen having empty inclusions, there is no interaction between the e-beam and the inclusion. Hence, inclusions do not give rise to a reduction of the signal and as a consequence air inclusions appear as the light regions in TEM images.

The detailed internal morphology of the electrospun PS fibers were investigated further using state of the art synchrotron radiation based X-ray tomographic microscopy (XTM). Since the X-ray imaging technique does not cause scattering at interfaces, as opposed to other visible wavelength-based techniques such as confocal laser microscopy, XTM is a unique technique to provide information on the internal structure of single fibers and beads. Fig. 3 shows representative XTM data. The voxel distribution through all gray scaled values is plotted in Fig. 3c. From these results internal pores are identified by a threshold of image and detection regions with a grayscale value between 30 and 89 . The isosurfaces of bead and fiber complexes shown in Fig. $3 \mathrm{a}$ and b, respectively, are reconstructed by connecting equi-gray scaled voxels with a value of 104. In Fig. 3d-f the gray scale distribution is depicted along the shown lines, sectioning either fibers or beads, where the results reveal the existence of internally less dense structures. The grayscale values from internal regions are not decreased to the grayscale level realized from voxels located in the air outside the electrospun features. It can be related to (a)

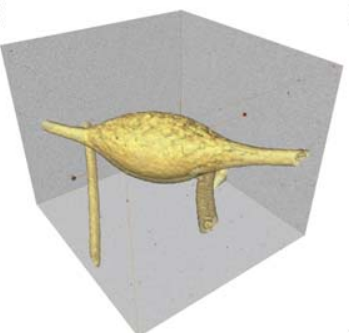

(b)
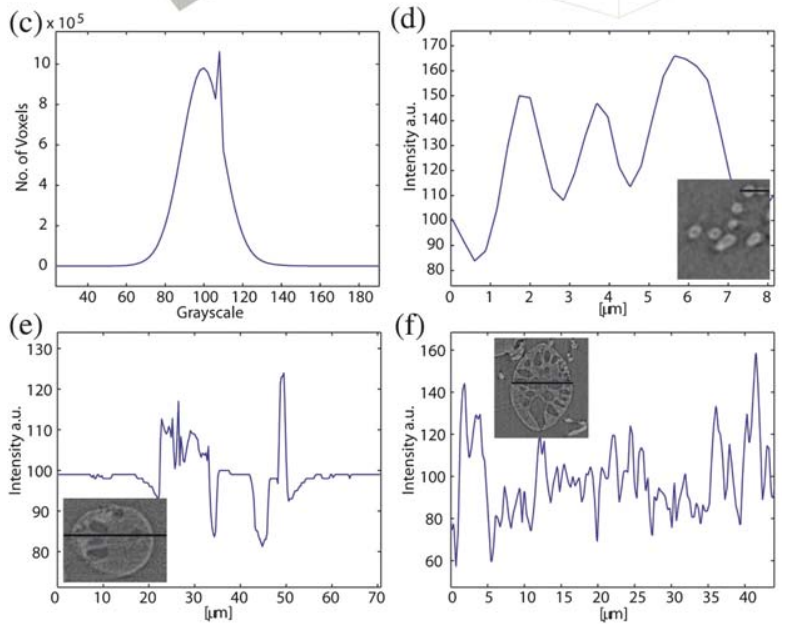

Fig. 3 Images in (a) and (b) show XTM reconstructions of electrospun PS beads and fibers obtained from PS solution in DMF at a concentration of $25 \%(\mathrm{w} / \mathrm{v})$. The grayscale distribution is shown in (c). The image cross-section in (b) is shown in (d) and (f) and the density variation is quantified along the indicated lines. The density variation shown in (e) is obtained by sectioning the bead shown in (a). (Note: the fiber diameters analysed by XTM are in the range of about 1 to 3 micron.)

noise collected during image acquisition or arise from an ultra-fine nanostructure which can be formed during electrospinning within these internally less dense regions. The ultra-fine nanostructure could consist of nanopores or an open network with branches below the resolution limit of the XTM technique. For structures above the resolution limit, XTM is still a unique nondestructive technique. Compared to other techniques, such as high resolution SEM or TEM, the visualization of covered internal morphologies in thick samples is not possible. Also sample preparation for these techniques is challenging when destruction of such ultra-fine polymeric nanostructures must be avoided. Our findings correlate with previous findings ${ }^{18-20}$ that during electrospinning internal porous structures in both fibers and beads can be formed. This can lead to a novel technique by which nanostructures can be fabricated to include internal functionalisation. The images reveal that it may be possible to tune the experimental parameters such as intra- and inter-fiber porosity that will enhance the functionality of fiber webs, where the local properties down to the scale of individual molecules are critical for the performance of the material, for example controlling the porosity of a tissue engineered scaffold, the location of individual filtration elements in a membrane, and controlled buoyancy.

\section{Conclusions}

We have presented the first internal morphology study of electrospun PS fibers using X-ray tomographic microscopy (XTM). The studies 
reveal that electrospun PS fibers have internal density variations and that the beads have internal porosities. The porous features of these electrospun PS fibers may be interesting in applications such as controlled buoyancy, controlled drug release and when the nanofibers are used in filtration/membrane applications. The results demonstrate XTM as a powerful technique to determine the internal complex morphologies. Recent developments in X-ray optics will provide new XTM setups with superior resolution and combined with image processing capabilities and such facilities will be a very interesting tool for quantifying complex internal structures of nanomaterials. $^{21}$

\section{Acknowledgements}

We gratefully acknowledge the funding to the project NanoNonwovens from The Danish Advanced Technology Foundation, the collaboration with Fibertex A/S, and the Danish Research Agency for the funding to the iNANO center. State Planning Organization (DPT) of Turkey is acknowledged for the support of UNAM-Institute of Materials Science and Nanotechnology through the National Nanotechnology Research Center Project. Dr Tamer Uyar acknowledges EU FP7-PEOPLE-2009-RG Marie Curie International Reintegration Grant (IRG) for funding NANOWEB (PIRG06-GA-2009-256428) project.

\section{Notes and references}

1 S. Ramakrishna, K. Fujihara, W.-E. Teo, T.-C. Lim and Z. Ma, An introduction to electrospinning and nanofibers, World Scientific, 2005, pp. $90-102$.

2 A. Greiner and J. H. Wendorff, Angew. Chem., Int. Ed., 2007, 46, 5670 .
3 D. Li and Y. Xia, Adv. Mater., 2004, 16, 1151.

4 F. Yao, L. Xu, B. Lin and G.-D. Fu, Nanoscale, 2010, 2, 1348.

5 K. Yoon, B. S. Hsiao and B. Chu, J. Mater. Chem., 2008, 18, 5326.

6 Z.-M. Huang, Y.-Z. Zhang, M. Kotaki and S. Ramakrishna, Compos. Sci. Technol., 2003, 63, 2223.

7 X-Ray Microscopy VII, ed. J. Susini, D. Joyeux and F. Polack, EDP Sciences, Paris, 2003.

8 W. Chao, E. Anderson, G. P. Denbeaux, B. Harteneck, J. A. Liddle, D. L. Olynick, A. L. Pearson, F. Salmassi, C. Y. Song and D. T. Attwood, Opt. Lett., 2003, 28, 2019.

9 L. Kipp, M. Skibowski, R. L. Johnson, R. Berndt, R. Adelung, S. Harm and R. Seemann, Nature, 2001, 414, 184.

10 J. Miao, P. Charalambous, J. Kirz and D. Sayre, Nature, 1999, 400, 342.

11 J. W. Miao, K. O. Hodgson, T. Ishikawa, C. A. Larabell, M. A. LeGros and Y. Nishino, Proc. Natl. Acad. Sci. U. S. A., 2003, 100, 110.

12 S. Marchesini, H. He, H. N. Chapman, S. P. Hau-Riege, A. Noy, M. R. Howells, U. Weierstall and J. C. H. Spence, Phys. Rev. B: Condens. Matter Mater. Phys., 2003, 68, 140101.

13 W. Chao, B. D. Harteneck, J. A. Liddle, E. H. Anderson and D. T. Attwood, Nature, 2005, 435, 1210.

14 J. H. Kinney, Annu. Rev. Mater. Sci., 1992, 22, 121.

15 P. C. J. Donoghue, S. Bengtson, X. Dong, N. J. Gostling, T. Huldtgren, J. A. Cunningham, C. Yin, Z. Yue, F. Peng and M. Stampanoni, Nature, 2006, 442, 680.

16 T. Uyar and F. Besenbacher, Polymer, 2008, 49, 5336.

17 T. Jarusuwannapoom, W. Hongroijanawiwat, S. Jitjaicham, L. Wannatong, M. Nithitanakul, C. Pattamaprom, P. Koombhongse, R. Rangkupan and P. Supaphol, Eur. Polym. J., 2005, 41, 409.

18 E. S. Megelski, J. S. Stephens, D. B. Chase and J. F. Rabolt, Macromolecules, 2002, 35, 8456.

19 J. Lin, B. Ding, J. Yu and Y. Hsieh, ACS Appl. Mater. Interfaces, 2010, 2(2), 521.

20 Y. Zhu, D. Yang and H. Ma, Nanoscale, 2010, 2, 910.

21 J. V. Nygaard, M. Ø. Andersen, K. A. Howard, M. Foss, C. Bünger, J. Kjems and F. Besenbacher, Biotechnol. Bioeng., 2008, 100, 820. 